\title{
Estrategias de evaluación empleadas por los docentes de Física en los contenidos de Estática a nivel de Educación Media
}

\author{
Assessment strategies used by Physics teachers in Statics content \\ at the Secondary Education level
}

\author{
Pedro Alejandro Blanco García
}

alejandrob145@gmail.com

Código ORCID: 0000-0003-2993-0730

Unidad Educativa Antonio Ricautte, Venezuela

Recibido: septiembre 2019 / Arbitrado: octubre 2019 / Publicado: enero 2020

RESUMEN

La física es una ciencia experimental que intenta dar respuestas a los fenómenos físicos observables, realizando las mediciones lo más certeras posibles; en este sentido, el docente facilitador de la asignatura debe proporcionar innovación con participación creativa y consciente en la formación del individuo, desde la peculiaridad de diseñar, ejecutar y evaluar actividades de enseñanza y aprendizaje en esta área. Por lo cual, el objetivo general fue describir las estrategias de evaluación empleadas por los docentes de física a nivel de Educación Media. El tipo fue descriptivo no experimental, se escogió un censo poblacional conformado por 26 docente pertenecientes al Municipio Carlos Arvelo. Se consideró aplicar una encuesta conformada por 18 ítems; cuya confiablidad fue calculada aplicando el coeficiente KuderRicharson. Al finalizar se concluyó, los docentes tienen conocimiento y usan en un $67,5 \%$ las estrategias de evaluación acordes con el proceso, pero en contra parte un 32,5\% no las aplica.

Palabras clave:

Estrategias de evaluación; estática; Educación Media

ABSTRACT

Physics is an experimental science that tries to give answers to observable physical phenomena, making the measurements as accurate as possible; In this sense, the teacher-facilitator of the subject must provide innovation with creative and conscious participation in the training of the individual, from the peculiarity of designing, executing and evaluating teaching and learning activities in this area. Therefore, the general objective was to describe the evaluation strategies used by physics teachers at the level of Secondary Education. The type was descriptive, not experimental, a population census consisting of 26 teachers belonging to the Carlos Arvelo Municipality was chosen. It was considered to apply a survey made up of 18 items; whose reliability was calculated applying the Kuder-Richarson coefficient. At the end it was concluded, the teachers have knowledge and use $67.5 \%$ of the evaluation strategies according to the process, but on the other hand $32.5 \%$ do not apply them.

Keywords: 


\section{INTRODUCCIÓN}

La educación según lo mencionan Montes, Peñaranda y Rodríguez (2012) es considerada como el proceso multidireccional mediante el cual se transmite conocimiento, valores, costumbres y formas de actuar a los seres humanos, para que de esta manera obtengan habilidades y destrezas que le permitan desenvolverse adecuadamente en el contexto social y educativo. A esto se le puede agregar que se aplica en un contexto específico, con la finalidad que el estudiante logre alcanzar objetivos sobre un contenido, en el que el docente es el guía que emplea estrategias y recursos que faciliten la obtención del conocimiento nuevo.

Uno de los componentes esenciales del proceso educativo mencionado es la evaluación educativa, ya que es el pilar fundamental para la verificación de los logros en el proceso de enseñanza y aprendizaje, desde sus inicios enmarcado en el positivismo clásico cuando se consideraba sinónimo de medición, de ahí al positivismo lógico, destacando la importancia del principio de la verificación, predefiniendo criterios que delimiten las proposiciones que se desean verificar empíricamente, hasta llegar al pospositivismo que está basado en el conocimiento objetivo, verificable intersubjetivamente con la ontología realista-crítica y aunado a lo empírico, con el conocimiento cualitativo que proporciona el contexto necesario para interpretar los datos cuantitativos.

Para ello se necesita integrar la función pedagógica de la evaluación que observa el desenvolvimiento, actuación, conducta, dentro y fuera del aula de clase, con el fin de precisar sus virtudes y defectos del estudiante visto en toda su esencia, de esta forma hacer los correctivos pertinentes de manera progresiva. Pero esto se logra cuando el docente cambie su actitud y aptitud en dirección a la evaluación desde una forma constructivista, desarrollando un pensar crítico, innovador, creativo, involucrándose en el ser, sentir de sus estudiantes al realizar la selección adecuada de la técnica e instrumentos de evaluación a utilizar para la apreciación de una situación determinada de aprendizaje. Cabe preguntarse si en Venezuela ¿se ha registrado un avance en materia de la evaluación? Salcedo (2010) al respecto afirma:

En cuanto a la evaluación en
Venezuela durante el período
estudiado (1914-2009), sólo ha
consistido, predominantemente, en
la elaboración y aplicación de
«exámenes» generalmente a juicio
del maestro o profesor, y según
normativas oficiales las cuales no
han sido objeto de estudio y
evaluación rigurosos, por lo que es
mucho lo que falta por hacer para
llegar a considerar esta disciplina
como una profesión con sus
criterios normativos, estándares y
regulaciones éticas. (p. 371)

El país se ha quedado estancado en lo que respecta a metodología de evaluación precisamente no por culpa de medidas gubernamentales, más bien por falta de concepción de la evaluación de los docentes, tal afirmación es reforzada cuando se menciona el problema de la evaluación en el subsistema de educación media en el documento de la Cámara Venezolana de Educación Privada (CAVEP, 2014).

Se genera una serie de reflexiones y críticas dirigidas a los tipos de evaluación, a los procedimientos empleados y a los resultados que se obtienen, los cuales aparecen asociados a los altísimos niveles de fracaso escolar en este nivel del sistema educativo. (p. 51)

Congruentemente Díaz y Hernández (2010) consideran el modo de la evaluación educativa empleadas por los docentes un factor de los rendimientos del alumnado, ya que "en el 
proceso educativo puede evaluarse prácticamente todo, lo cual implica aprendizaje, enseñanza, acción docente, contexto físico, y educativo, programas, currículo, aspectos institucionales, entre otros aspectos" (p. 306).

Para que se haga con eficiencia el proceso educativo se debe contar con referentes teóricos en la materia evaluativa que orienten a la elaboración y uso de determinados criterios de evaluación para poder obtener una representación lo más fidedigna posible, lo cual actualmente muchos docentes emplean la praxis evaluativa sin referentes teóricos, $y$, al desempeñar sus funciones en alguna institución educativa, su concepción del modo que aprenden y se enseña es muy poca e igualmente su concepción sobre ¿qué, cómo y cuándo evaluar? Donde su enseñanza es meramente verbalista-expositiva y unidireccional, su evaluación se centra solo en los resultados de aprendizaje y no en los procesos, enfatizando demasiado la función social de la educación, y en particular, la evaluación sumativa-acreditativa. Cuentan con un conocimiento más o menos preciso de una serie de estrategias, instrumentos y técnicas para evaluar aprendidos en distinta experiencias pedagógicas, pero demuestra una escasa reflexión sobre ellas (Díaz y Hernández, 2010).

Demostrando en muchas ocasiones que malinterpretan las funciones de la evaluación, no dándole el lugar que le corresponde a la función pedagógica para la regulación de los procesos de enseñanza, hasta el punto de anularla, por no ponerle limite a la función social.

Tomando en cuenta que la física es una ciencia experimental que tiene como finalidad la explicación de lo que sucede cuando se producen cambios en la materia y sus propiedades, los docentes de física en su ejercicio en tercer año de Educación Media emplean frecuentemente la evaluación cuantitativa, como lo es el examen con lápiz y papel, conllevando a un aprendizaje memorístico, por repetición, y en el caso de la enseñanza de los procesos de resolución de problemas, enseñan de manera mecánica y trivial, enfocados en solo utilizar ecuaciones y más ecuaciones, en vez de enseñarle de manera metodológica que le permitan tener estrategias clara y sencillas que aborden los problemas en busca de solución.

Esto se debe a los profesionales que imparten esta asignatura, pues muchas veces no son egresados en esta especialidad, ocasionando un declive en los resultados, por falta del dominio de los contenidos, planeando sus clases solo para cubrir un mero requisito, conllevando a una evaluación tradicional, pues no estima con mayor objetividad los indicadores deseados que logren los estudiante, llevando a altos índices de reprobación, deserción y repetición de la materia.

En atención a lo que se ha explicado, se es necesario comprobar el empleo de estrategias evaluativas por los docentes de física en el nivel de Educación Media, pertenecientes al Municipio Carlos Arvelo. De lo expresado anteriormente se logró ejecutar los siguientes objetivos específicos: Describir las estrategias de evaluación empleadas por los docentes de física de tercer año de Educación Media. Para alcanzar el mismo fue necesario cumplir con los objetivos específicos: 1. Diagnosticar las estrategias de enseñanza aplicadas por los docentes a la evaluación de los contenidos de física de tercer año de educación media general. 2. Identificar las técnicas usados por el docente de física, y 3. Determinar los instrumentos utilizadas por el docente.

Por otro lado, los principales beneficiarios son los docentes y estudiantes al proporcionar actividades de creación y aplicación donde se logren evaluar habilidades, actitudes y combinaciones de conocimiento que necesite aplicar en la vida real, asumiendo como meta, la actividad de autoevaluación, capacitando al alumno como ser competente. Además, la comunidad también tendrá beneficios, ya que la educación será favorecida y mejorará su calidad 
de enseñanza a los ciudadanos, repercutiendo directamente a favor de ella.

Como un antecedente relevante a este estudio se tiene a Peraza y Camargo (2015), en su investigación titulada "Estrategia de evaluación utilizados por los docentes de Física en el Tercer Año de Media General del Distrito Escolar № 8 del Municipio Mario Briceño Iragorry del estado Aragua fundamentado en el enfoque Díaz y Hernández". Los resultados obtenidos demostraron que la mayoría de los docentes conocen algunas técnicas e instrumentos que llevan a cabo en el desarrollo de ella, sin embargo, los docentes presentan fallas en cuanto a diversas técnicas e instrumentos utilizadas en el mecanismo de evaluación.

Con referencia a lo anterior, según considerar la evaluación desde una perspectiva constructivista, es tratar de reflexionar sobre el proceso de enseñanza y aprendizaje, destacando primordialmente decisiones pedagógicas, la cual promueve una enseñanza se adapte a la diversidad del alumnado. Promueve el aprendizaje con sentido y tenga un valor funcional para los alumnos, que permita la regulación de la enseñanza y aprendizaje, teniendo como meta la autorregulación de los alumnos en la evaluación (Díaz y Hernández, 2010). Para ello, el docente cuanta con diversidad de estrategias y procedimientos que le permiten obtener información valiosa que determina de la manera más fidedigna posible con criterios bien definidos un juicio valorativo que interpreta el cómo y hasta qué punto se alcanzaron los criterios de aprendizajes.

\section{a) La evaluación en el proceso de enseñanza y aprendizaje}

La asociación primordial cuando se menciona la palabra "evaluación" es considerada medición sobre la importancia de las características de un objeto, hecho o situación particular, la cual además, incluyendo estimaciones cualitativa y cuantitativa. En cambio, en la evaluación en el proceso de enseñanza y aprendizaje se anexa a esta concepción el mecanismo que controla, regula y conoce los factores y problemas que promueven $u$ obstaculiza este proceso, aportándonos argumentos para proponer correcciones y mejoras de los resultados.

A lo que se refiere a las inquietudes, dudas y ayuda que expresan los profesores en cursos de formación o asesorías, en ella se registra la necesidad de entender el qué, cuándo y para qué evaluar, así como los procedimientos, técnicas, instrumentos y criterios a utilizar en las actividades de evaluación. Donde se percata que una conlleva a la otra, ya que la reflexión del qué hay que evaluar, para qué evaluar y cuándo evaluar, guiará de manera eficiente para la tomas de decisiones respecto a los instrumentos o procedimientos y el diseño y el planteamiento de actividades que se utilizan en la materia evaluativa. Esta actividad evaluativa se debe realizar tomando en cuenta no solo el aprendizaje de los alumnos, sino también las actividades de enseñanza que realiza el docente y la relación con los aprendizajes.

\section{b) Directrices para una evaluación constructivista}

En los últimos años han aparecidos distintas aproximaciones y paradigmas sobre evaluación educativa. Pero solamente este desmesurado interés prevalece solo en los productos observables del aprendizaje de todo un proceso de construcción del conocimiento escolar.

La evaluación debe atender a todo el proceso constructivo del aprendizaje, aquellas actividades constructivas y elaboraciones de determinadas representaciones (esquemas, significados) sobre los contenidos se consideran recursos cognitivos y afectivos que los alumnos utilizan, que mediante técnicas e instrumentos se obtendrán información valiosa del proceso de construcción del conocimiento. Para evaluar este proceso de construcción se es necesario apreciar las distintas fases del proceso, por 
ejemplo, la naturaleza y complejidad de los esquemas previos existente, el grado de control y responsabilidad que los alumnos alcanzan respecto al aprendizaje mediante el uso de sus propios recursos cognitivos e interpretaciones significativas, y mediante las observaciones continuas captar la naturaleza de los errores y dificultad encontradas en el proceso de construcción.

Así como el aprender significativamente es aprender a aprender y aprender a autorregularse, y son consideradas metas en la educación, la actividad de aprender a autoevaluarse debe ser considerada igualmente relevante. Cabe agregar entonces, es necesario capacitar al alumno como un aprendiz competente, proponiendo situaciones y espacios para tal práctica, antes el debido preaviso de criterios que ellos aprenderán para las valoraciones.

Una práctica desafortunada, pero que se ha hecho costumbre entre el profesorado de casi todos los niveles educativos, consiste en establecer una marcada distancia entre lo que se enseña y lo que luego se evalúa. Para evitar este comportamiento, hay que plantear tareas, actividades e instrumentos de evaluación que demanden $\mathrm{o}$ impliquen las interpretaciones y significados construidos, añadiéndoles actividades didácticas para que el alumno reconozca y valoren la utilidad de aprender comprendiendo.

Las estrategias de enseñanza son medios o recursos para prestar la ayuda pedagógica ajustada a las necesidades de progreso de la actividad constructiva de los estudiantes de forma reflexiva y flexible para promover el logro de aprendizajes significativos, considerando que las estrategias de enseñanza que se presentan son complementadas por las estrategias evaluativas, además de la motivacionales, las de trabajo cooperativo o colaborativo, entre otras, permitiendo el enriquecimiento del proceso didáctico, ya que al enseñar, y evaluar se logra confirmar los conocimientos e reorientar el proceso de enseñanza, por ello se estará explicando las estrategias para activar y usar los conocimientos previos, y para generar expectativas apropiadas en los estudiantes, estrategias para ayudar a organizar la información nueva por aprender, estrategias para promover una enseñanza situada.

Para atender de la mejor manera posible el problema de qué enseñar, se debe buscar de manera eficiente y reflexiva como lograr a través de determinadas estrategias de enseñanza que el alumno tenga un desarrollo personal, que se en culturicen y participen de una forma activa y critica en su contexto, por ello la insistencia de que el docente debe poseer un bagaje amplio de estrategias, además de conocer su función, para que se utilizan y como se les puede sacar mayor provecho. De hecho, varias de las estrategias de enseñanza que presentamos aquí, pueden ser utilizadas pensando en un doble objetivo: el primero, ya antes mencionado, para promover aprendizajes significativos, lo cual ya de por si tiene un valor pedagógico indiscutible, y el segundo para introducir y enseñar a los alumnos como elaborarlas y posteriormente, con las ayudas (modelamiento, explicaciones, ejercitaciones apropiadas) dar paso a que ellos las puedan aprender y utilizar como estrategias de aprendizaje.

Con respecto a las estrategias para activar y usar los conocimientos previos, y para generar expectativas apropiadas en los estudiantes es necesario incluir todas aquellas estrategias que están dirigidas a activar o generar los conocimientos previos en los aprendices. Simple y sencillamente la actividad constructiva no sería posible sin conocimientos previos que permitan entender, asimilar e interpretar la información nueva para luego, por medio de ella, reestructurarse y transformarse hacia nuevos posibles. De ahí la importancia de activar los conocimientos previos pertinentes de los estudiantes, con el fin de retomarlos y relacionarlos con momentos adecuados a la información nueva por aprender que se descubre 
o construye de manera conjunta con los estudiantes. Las estrategias que se presentan en este apartado, preferiblemente deberán emplearse al inicio de cualquier secuencia didáctica, o bien antes que los aprendices inicien cualquier tipo de actividad de indagación, discusión o integración sobre el material de aprendizaje propiamente dicho, sea por vía individual o colaborativa.

Entre las estrategias que se pueden emplear en tal sentido, se presentan aquí las que han demostrados ser más efectivas, a saber: la actividad focal introductoria, las discusiones guiadas y las actividades generadoras de información previa. También se presenta por la similitud con estás los objetivos o intenciones educativas, para generar en los estudiantes expectativas de aprendizaje apropiadas y ayudarles a atribuir sentido a los aprendizajes próximos.

En la aplicación de esta estrategia los estudiantes desde el inicio activan sus conocimientos previos, y gracias a los intercambios en la discusión con el profesor pueden desarrollar y compartir con sus compañeros de forma espontánea conocimiento y experiencias previas que pudieran no poseer (o al menos no del mismo modo) antes de que la estrategia fuese iniciada.

Se debe considerar en el momento de planificar y aplicar una discusión lo siguiente: tener claro los objetivos de la discusión y hacia dónde se le quiere conducir; de este modo se podrá activar y favorecer la compartición de conocimientos previos pertinentes, para el aprendizaje de los nuevos contenidos que se abordaran posteriormente; introducir la temática central del nuevo contenido de aprendizaje y solicitar a los estudiantes que expongan lo que saben de esta. Es pertinente animar a participar a una buena cantidad de estudiantes del grupo-clase desde el inicio, para la discusión, se recomienda elaborar preguntas abiertas que requieran más de una respuesta afirmativa o negativa, no solo se debe conducir la discusión sino también participar en ella y modelar la forma de hacer preguntas y dar respuestas

Tanto la discusión guiada como la actividad generadora de información previa deben ser breves y se les debe considerar como recursos estratégicos útiles para los fines ya mencionados. La influencia de tales estrategias no termina cuando estas concluyen, ya que pueden ser retomadas durante la secuencia didáctica en varias ocasiones como "marcos referenciales que ya se han compartido", para ayudar a comprender las explicaciones o actividades que se añaden sobre la marcha

Ampliamente utilizados como recursos didácticos, los organizadores gráficos pueden definirse como representaciones visuales que comunican la estructura lógica del material instruccional que va a aprenderse. Son de gran utilidad cuando se quiere resumir u organizar corpus significativos de conocimiento y pueden emplearse como estrategias de enseñanza, o bien puede enseñarse a los estudiantes a utilizarlo como estrategias de aprendizaje. Su efectividad ha sido ampliamente comprobada en ambos caso para la mejora de los procesos de recuerdo, comprensión y aprendizaje.

Por otra parte los mapas conceptuales son representaciones graficas de segmentos de información o conocimiento de tipo declarativo. Como estrategias de enseñanza, pueden representarse temáticas de una disciplina científica, programas de curso o currículos y hasta utilizarlos como apoyo para realizar procesos de negociación de significados en la situación de enseñanza (compartir y discutir con los estudiantes los contenidos curriculares que están aprendiendo).

Sin embargo los cuadros sinópticos, proporcionan una estructura coherente global de una temática y sus múltiples relaciones. Organiza la información sobre uno o varios temas centrales que forman parte de la temática 
que interesan enseñar. Los cuadros sinópticos son generalmente bidimensionales y están estructurados por columnas y filas. Cada columna y/o fila debe tener una etiqueta que representa una idea o concepto principal. Las columnas y filas se cruzan $y$, en consecuencia, se forman celdas que son llenados con los distintos tipos de información. Esta información puede estar constituida por hechos, ejemplos, conceptos, principios, observaciones, descripciones, explicaciones, proceso, e incluso pueden colocarse ilustraciones de diversos tipos.

Por otro lado, el uso de las líneas de tiempo, las cuales son representaciones graficas que permiten organizar y visualizar eventos o hitos dentro un continuo temporal. Son muy útiles para la enseñanza de conocimiento histórico porque por medio de esta se pueden observar y representar visualmente las relaciones de anterioridad y posterioridad entre eventos o acontecimientos, comprender las unidades de mediadas y los intervalos temporales, la noción de sincronicidad y diacronicidad dentro de un periodo histórico y pueden dar paso al entendimiento de la casualidad histórica.

Desde una perspectiva socioconstructivista, la enseñanza situada puede definirse como aquella propuesta pedagógica que se diseñan y estructura con la intención de promover aprendizaje situados, experienciales y auténticos en los estudiantes, que les permita desarrollar habilidades y competencias muy similares o iguales a las que se encontraran en situaciones de la vida cotidiana o profesional Algunas propuestas pedagógicas por sus características propias pueden incluirse dentro de ella, son el denominado aprendizaje basado en problemas, el aprendizaje basado en análisis y estudios de casos y el aprendizaje mediantes proyectos.

Con todo ello se busca conseguir la construcción del conocimiento personal y particularmente la construcción conjunta con los compañeros y con el enseñante quien guía y supervisa todo el proceso. Se dice que pueden desarrollarse y participarse distintos tipos de habilidades cognitivas, expositivas, comunicativa y de pensamiento crítico y al mismo tiempo pueden aprender los contenidos de los programas escolares que éstos se inserten. La puesta en marcha de la metodologías puede acompañarse de una estrategia de evaluación autentica tal como u portafolio en el que se planifica con los estudiantes la colección estratégica de distintos tipos de muestras y vestigios correspondientes a cada una de las fases que componen dichas metodologías para valorar los progresos.

Por su parte el interés del profesor al evaluar los aprendizajes debe estar enfocado en que condición los alumnos han construido interpretaciones significativas hacia los contenidos revisados, además del grado de atribución a su sentido y valor funcional, la relación a la utilidad que estos aprendizajes puedan tener para su futuro. Por ello, la finalidad es desear que el aprendiz, logre ciertos aprendizajes significativos, construya un conocimiento que le permita saber que sabe, como lo sabe y en qué y para que contexto le puede resultar útil eso que sabe. A mayor cantidad y diversidad posible de indicadores e instrumento para poder estimar con mayor objetividad el proceso de construcción y el producto construido de aprendizajes significativo.

Durante la función pedagógica se evalúan los procesos para obtener información que permitan en un momento determinado saber que paso con el aprendizaje de los estudiantes y con la respectivas estrategias de enseñanza, para que en ambos caso, puedan hacerse las mejoras y ajustes necesario. En consecuencia esta función pedagógica es un asunto central para la confección de una enseñanza verdaderamente adaptativa. Ampliando un poco más, puede decirse que la función pedagógica se integra al proceso de enseñanza, como una genuina evaluación continua. Está dirigida a tomar decisiones de índole pedagógica y verdaderamente justifica o le da sentido a la 
evaluación. Es por eso que los objetos de la evaluación no deben ser solo los procesos de aprendizaje de los estudiantes, sino también las prácticas y estrategias de enseñanza.

Cabe agregar entonces, existen diversas propuestas de clasificación de la evaluación del proceso de enseñanza y aprendizaje, en esta sección estudiaremos la que desde hace ya varias décadas se ha incorporado al discurso docente, es valiosa y de gran aporte. Esto se refiere a la clasificación que distingue a los tipos de evaluación por el momento en que son introducidos en un determinado episodio, proceso o cielo educativo. Estas tres clases de evaluación son las llamadas: diagnóstica, formativa y sumativa.

Con respecto a la evaluación diagnóstica, esta se caracteriza por realizarse previamente al desarrollo del proceso educativo, se clasifica en dos tipos: inicial y puntual. La primera atiende exclusivamente antes de algún proceso o ciclo educativo amplio, con el objetivo de reconocer si los alumnos poseen una serie de conocimientos previos pertinentes que le permitan asimilar $y$ comprender los nuevos saberes que se les presentarán, obteniendo información que indica qué grado de adecuación de la capacidades cognitivas en relación con el programa en consecuencia hacer los ajustes didácticos necesarios que permitan elevar a un grado mayor de significatividad psicológica y lógica los contenidos.

Por otro lado, la evaluación formativa se realiza simultáneamente con el proceso de enseñanza y aprendizaje y es considerada, más que las otras, como una parte reguladora $y$ consustancial del proceso. Su función es pedagógica, se encarga de regular el proceso de enseñanza y aprendizaje, de esta forma adaptar o ajustar las condiciones pedagógicas (estrategias, actividades, etcétera) en favor del aprendizaje de los alumnos. Se preconcibe de la idea de supervisar el proceso del aprendizaje considerándola una actividad continua de reestructuraciones producto de las acciones del alumno y de la propuesta pedagógica.

Y por último la evaluación sumativa, en la cual es considerada como la evaluación por antonomasia, al punto que cuando se habla de evaluación en las comunidades escolares inmediatamente se le asocia con ella; este tipo de evaluación también es denominada evaluación final, por ser la que se realiza al término de un proceso o ciclo educativo cualquiera. A través de esta, el docente puede conocer si los aprendizajes estipulados en las intenciones se cumplieron según los criterios y las condiciones expresadas en ellas. Especialmente la evaluación sumativa provee información que permite derivar conclusiones importantes sobre el grado de éxito y eficacia de la experiencia educativa global emprendida.

No obstante, la evaluación como proceso es entendida de ese modo, ya que proporciona ajustar la ayuda mediante comprobación constante de las vicisitudes del proceso de construcción de conocimiento y modificar la acción educativa en consecuencia, realizándose a lo largo de todo el proceso de enseñanza y aprendizaje. Es integral pues en el marco de interpretación constructivista interesa el grado en que los alumnos han construido, por medio de la ayuda pedagógica recibida, creando interpretaciones significativas las que les atribuyen sentido y valor funcional.

Para la evaluación del proceso existen tres momentos: la autoevaluación: que es la evaluación del alumno acerca de sus propias producciones, la coevaluación: la evaluación de un producto del alumno no realizada por él en conjunción con el docente (o con otro alumno), y la heteroevaluación es la evaluación donde el docente es el único y exclusivo agente evaluador.

\section{MÉTODO}

El presente estudio se enmarca dentro de una investigación de carácter descriptivo no experimental, en este sentido Hernández, et al. 
(2014) señalan "los estudios descriptivos se busca especificar las propiedades, características y los perfiles de personas, grupos, comunidades, procesos, objetos o cualquier otro fenómeno que se someta a un análisis" (p. 92). Englobando, pretende medir la información recogida con el fin de establecer una descripción de su estructura o comportamiento de la situación planteada.

El tipo de diseño expuesto en esta investigación estuvo fijado en la modalidad de campo y transeccional, con apoyo de bases documentales, es de campo por que la recolección de datos se obtuvo directamente de la realidad donde ocurren los hechos. Para que se cercioré las condiciones reales en que se han conseguidos los datos.

Para el desarrollo de esta investigación, se consideró una población de veintiséis (26) docentes de física perteneciente al Municipio Escolar 2.1, Guigue, Carlos Arvelo; los cuales fueron diez (10) docente de física de 3er año.

La técnica que se utilizó fue la encuesta, la cual se aplicó a los docentes de física del municipio antes mencionado, a través de un cuestionario compuesto por dieciocho (18) ítems dividido en tres (3) dimensiones: estrategias de enseñanza, técnicas de evaluación e instrumentos de evaluación, cuyas respuestas fueron dicotómicas siendo las opciones sí o no con argumento.

Por otro lado, se utilizó un instrumento ya validado por los autores Peraza y Camargo (2015), en su trabajo titulado "Estrategias de evaluación utilizadas por los docentes de física en el tercer año de Educación Media General del Distrito Escolar № 8 del Municipio Mario Briceño Iragorry del Estado Aragua fundamentado en el enfoque de Díaz y Hernández".

\section{RESULTADOS}

Los resultados se presentaron según la dimensión. Primeramente la relacionada a Estrategia de Enseñanza, donde un 72,7\% contestó que si utilizan las discusiones guiadas para generar los conocimientos previos en los estudiantes. El 81,8\% concibe las discusiones al inicio del contenido, un 90,9\% de los docentes aplican la estrategia de lluvias de ideas para relacionar los contenidos adquiridos por el estudiante con el conocimiento nuevo a impartir, el 54,5\% utilizan el mapa conceptual para la comprensión de la teoría del contenido de estática, en el caso de la organización 63,6\% ejecutan el cuadro sinóptico en el contenido de física para la comprensión clara y sencilla, por último el 54,5\% realizan la línea de tiempo como estrategia de enseñanza. En los comentarios el $27,2 \%$ si con argumento, $35,1 \%$ si $\sin$ argumento, 5,2 no con argumento y $32,4 \%$ no sin argumento. En conclusión se observó que $67,5 \%$ si realizan estrategia de enseñanza y $32,5 \%$ no lo aplican.

En lo referente a la dimensión Técnicas de Evaluación, se observó que un 90,9\% de los docentes utilizan la observación para determinar el proceso de aprendizaje en el aula consolidado en los estudiantes en los contenidos de física, en cuanto al momento de aplicar la evaluación el $63,6 \%$ utilizan la observación en todo los contenidos de física, el 72,7\% aseguran realizar prueba abierta y cerradas, sin embargo se observó un elevado $81,8 \%$ que no realiza la evaluación de portafolio como cierre del contenido y un $63,6 \%$ respondió de manera positiva para realizar prueba de ensayo como evaluación. En cuanto a los comentarios se observó que el 36,3\% si con argumento, 31,8\% si sin argumento, 3\% no con argumento y $33,3 \%$ para no sin argumento. En conclusión se observó un $63,6 \%$ que aplica técnicas de evaluación y un 45,1 que no aplica.

En lo referente a la Dimensión Instrumentos de Evaluación, se observó que un 54,5\% de los docentes no utilizan las rubricas como instrumento de evaluación, en contraste se observó que el 81,8\% afirman utilizar la escala de valorización para comprobar aspectos importantes en el proceso de aprendizaje en los contenidos de física, un 54,5\% asegura no llevar 
un registro anecdótico del comportamiento de los estudiantes, en cambio el $54,5 \%$ de los docentes plasman la experiencia de los estudiantes en el aula en un diario de clase, por último un elevado $72,7 \%$ asevero garantizar la obtención de los conocimientos del contenidos aplicando la lista de verificación. En los comentarios se observa un $27,3 \%$ sí con argumento, 32,7\% sí sin argumento, el 9,1 no con argumento y $30,9 \%$ no sin argumento. En conclusión se observó un 60\% que aplican instrumentos de evaluación y un $40 \%$ que no emplean.

En lo referente a las dimensiones de estrategia de Enseñanza, técnica e instrumento de evaluación se comprobó que el $62,1 \%$ sí la utilizan, sin embargo $37,8 \%$ no la manejan, también se observó que en la dimensión estrategias de enseñanza el ítem 1 con $72,7 \%$, el 2 con $81,8 \%$ y finalmente ítem 3 90,9\% muestran respuestas altas afirmativas, en contraparte los ítems № 4,6 y 7. Un 45,5\% no lo utilizan, un 90,9\% del ítem № 8 confirman usar las técnicas de evaluación y un $81,8 \%$ del ítem № 12 no la ejecuta, un $72,7 \%$ del ítem 18 aseguran utilizar los instrumentos de evaluación y un $54,5 \%$ de los ítems № 14 y 16 no la utilizan. En cuanto a los comentarios el 30,3\% de los encuestados lo utilizan con argumentos, un $33,3 \%$ que aseguran utilizarla pero justifican, además un 5,5\% manifiestan no usarla con argumento y un $32,3 \%$ no sin justificación. Destacando que existe la tendencia del 90,9\% siendo el valor más alto en las dimensiones estrategias de enseñanza y técnica de evaluación, siendo solo el 32,3\% que argumenta por qué la utilizan y un $33,3 \%$ que la usan sin argumentar.

\section{CONCLUSIONES}

Una vez analizados los resultados de la investigación, contrastados con los teóricos y atendiendo a los objetivos anteriormente planteados se llegó a la siguiente conclusión: se logró confirmar que algunos de los docentes de física tienen conocimiento de estrategias de evaluación. Tomando en cuenta los resultados obtenidos mediante el cuestionario se observa que la mayoría de los docentes aplican las estrategias de enseñanza al momento de dar la clase. En relación al primer objetivo específico diagnosticar las estrategias de enseñanza utilizadas por los docentes, los resultados arrojan que los docentes tienen conocimiento y usan en un $67,5 \%$ las estrategias de enseñanza, pero en contra parte un $32,5 \%$ no las aplica. Es recomendable tomar medidas para añadir nuevas estrategias de enseñanzas propuestas por autores constructivistas que incentiven el aprendizaje significativo en los estudiantes, aportando un cambio a la manera tradicional y tediosa que se tienen de aplicar monótonamente estrategia de evaluación que ocasiona fatiga, sustituyendo con por actividades que interactúen el proceso de enseñanza y aprendizaje que fomenten una actitud de participación convirtiéndose en actividades entretenidas, novedosas y consolidando un pensar crítico, lógico y creativo en los estudiantes.

En relación al segundo objetivo específico: Identificar las técnicas usados por el docente de física de tercer año de Educación Media, se puede concluir que un elevado $63,6 \%$ emplean técnica de evaluación en contra parte un $45,1 \%$ que no la aplica, observando que los resultados muestran que los docentes se inclinan hacia la utilización de diversas técnicas de evaluación, para el logro de la consolidación de conocimientos de la asignatura es recomendable valorar el aprendizaje tanto en el proceso como el resultado, de esta manera obtener los objetivos de la evaluación.

Finalmente, el último objetivo de esta investigación destinado a determinar los instrumentos utilizados por los docentes de física de tercer año de educación media general. Se observó que el 60\% hacen uso de los instrumentos de evaluación en contra parte un $40 \%$ no aplica. La implementación de variados instrumento de evaluación facilita la obtención de mejores resultados. 


\section{REFERENCIAS}

CAVEP (2014) La Transformación del Sistema Educativo Venezolano. Recuperado de https://www.google.co.ve/url?sa=t\&rct=j\&q $=\&$ esrc $=s \&$ source $=$ web $\& c d=1 \& c a d=r j a \&$ uact $=8 \&$ ved $=0$ ahUKEwiLXsSJgoTTAhXG6yYKHXTH D6QQFggaMAA\&url=http\%3A\%2F\%2Fwww.c erpe.org.ve\%2Ftl_files\%2FCerpe\%2Fconteni do\%2Fdocumentos\%2FCalidad\%2520Educati va\%2FPropuesta\%2520Cavep $\% 2520$ al\%2520 ME\%2520(2).pdf\&usg=AFQjCNGm9eFpxmgk rnP16inCDn9HmKu1uQ\&sig2=vlGMe5LEvS91 $-m \times W B n M W 2 Q$

Díaz, F. y Hernández, G. (2010). Estrategias docentes para un aprendizaje significativo (3a ed.). México, D.F., México: McGraw-Hill Interamericana

Hernández, R., Fernández, C. y Baptista, P. (2014). Metodología de la investigación (6a ed.). México, D.F., México: McGraw-Hill Interamericana

Montes, A., Peñaranda, K; y Rodríguez, M. (2012). Validación de un propuesta didáctica para la enseñanza del contenido de electrostática, centrada en los estilos de aprendizaje de los estudiantes que cursan electromagnetismo I en la Universidad Pedagógica Experimental Libertador del Instituto Pedagógico de Barquisimeto "Luis Beltrán Prieto Figueroa". Recuperado de http://files.integraciondesaberes.webnode.c om.ve/200000081-

4de524edf2/validaci\%C3\%B3n\%20de\%20un a\%20propuesta\%20didactica\%20para\%20la \%20ense\%C3\%B1anza\%20de\%20la\%20electr ostatica.pdf

Peraza, M. y Camargo, B. (2015). Estrategias de evaluación utilizadas por los docentes de física en el tercer año de educación media general del distrito escolar $\mathrm{n}$ - 8 del municipio Mario Briceño Iragorry del estado Aragua fundamentado en el enfoque de Díaz y Hernández. Recuperado de http://www.riuc.bc.uc.edu.ve/handle/12345 $6789 / 2692$

Salcedo, G. (2010). La evaluación educativa y su desarrollo como disciplina y profesión: presencia en Venezuela. Revista de Pedagogía, 31 (8), 331-378 